\title{
Optical Flares: Observations and Interpretations
}

\author{
L.V. Mirzoyan \\ Byurakan Astrophysical Observatory, Armenia
}

\begin{abstract}
Observational results on flare stars in the Galaxy and their interpretation, based on the observational approach to early stages of stellar evolution at Byurakan, are considered. The evolutionary path of red flare stars is determined. Stellar flare activity is explained as an internal phenomenon of stars. The significance of photographic observations of stellar flares in star clusters and associations is emphasized.
\end{abstract}

\section{Introduction}

The discovery and study of red dwarf flare stars in the solar vicinity and in star clusters and associations had a fundamental significance for the physics and evolution of red dwarf stars. Flare stars were discovered in the 1950s in the solar vicinity and named UV Cet type variables. The quiescent radiation of these stars is interrupted by randomly distributed flares. UV Cet stars have very low luminosities and are seen in the vicinity of the Sun. The total number of known UV Cet stars is at present about 150 (Shakhovskaya 1995).

Ambartsumian (1954) noted that UV Cet stars at minimum light are similar to normal red dwarfs, but during flares exhibit some peculiarities of the $\mathrm{T}$ Tau stars, the characteristic population of stellar associations. This suggests a common nature of UV Cet and T Tau stars, an idea which was strengthened by the discovery of flare stars of higher luminosity in the Orion association (Haro \& Morgan 1953). The existence of luminous young flare stars, also in the Monoceros and Taurus Dark Cloud associations, indicates a bridge between the UV Cet and the T Tau stars. Haro (1957) suggested that the stage of flare activity is an evolutionary stage of red dwarfs which follows the T Tau stage. Ambartsumian (1970) found that this path of evolution is the only possible one for all red dwarf stars.

Photoelectric observations of UV Cet stars allow us to study flare events in detail. Photographic observations of stellar flares in clusters and associations, in spite of the low time resolution, turned out to be essential for the study of red dwarf stars in large numbers. We here consider the results of optical observations of stellar flares and their interpretation from the point of view of physics and evolution of red dwarf stars. It is based mainly on investigations carried out at the Byurakan Astrophysical Observatory. 


\section{Photographic observations of flares in star clusters and associations}

After Ambartsumian (1969) had shown that all low luminosity stars in the Pleiades must be flare stars, numerous photographic observations of stellar flares in regions of star clusters and associations were made with wide-field telescopes at the Tonantzintla, Asiago and Byurakan, and subsequently also at the Abastumani, Konkoly and Rozhen observatories. Photography with wide-field telescopes allows to detect all sufficiently powerful stellar flares which occur during the exposure time in a given field.

The search for stellar flares by photographic observations is usually carried out by the method of stellar chains. The exposure time for each image in a stellar chain is 5-15 minutes, depending on the spectral band of observation. The analysis of photoelectric observations of UV Cet flares by Moffett (1974) shows that out of 297 flares registered in the $U$-band, only 21 , and of 342 flares in the $B$-band, none could have been detected by photographic observations with an exposure time of 5 minutes for each image due to short duration and/or low amplitude of the flares.

Photographic flare observations permit the detection of flares with amplitudes larger than $0{ }^{\mathrm{m}} 5$. The efficiency of photographic observations of flares in regions of star clusters and associations is due to the wide field of the telescope, which monitors the large number of flare stars concentrated in these areas. The probability of observing a flare in a given flare star in the Pleiades with a $1-\mathrm{m}$ Schmidt telescope may be as large as $1 / 1000$ per hour. Because of the large number of flare stars in this region, a flare is detected usually every 1-2 hours on photographic plates (Mirzoyan 1981).

\section{Light curves and amplitudes of stellar flares}

Because of the low time resolution, photographic light curves of flares are distorted and can give only a general impression (Mirzoyan 1980). The amplitude of a flare increases towards shorter wavelengths. As a consequence, the effectivity of flare observations for fairly bright stars is largest in the $U$-band. For stars near or below the magnitude limit of photographic observations in the UV, it can be higher in the $B$-band. Results of photographic flare observations at Byurakan Observatory in regions of star clusters and associations (Table 1) show that they were actually more effective in the $B$ - than in the $U$-band.

Table 1. Effectivity of photographic observations by the method of stellar chains in the Pleiades region, carried out with the $1 \mathrm{~m}$-Schmidt telescope of the Byurakan Observatory in $1967-1976$ (Mirzoyan 1981).

\begin{tabular}{lcc}
\hline Spectral band & $B$ & $U$ \\
\hline Duration of observations [h] & 224 & 202 \\
Number of detected flares & 127 & 85 \\
Mean number of flares/hour & 0.57 & 0.42 \\
\hline
\end{tabular}




\section{Colours of stellar flares}

Photographic colour indices $B-V$ and $U-B$ of stellar flares by the chain method were obtained in 1979-1981 (Mirzoyan et al. 1981, 1983) using three wide-field telescopes: the $1 \mathrm{~m}$ and $0.53 \mathrm{~m}$ Schmidt cameras of the Byurakan Observatory (in $U$ and $B$, respectively) and the $0.70 \mathrm{~m}$ Maksutov telescope of the Abastumani Observatory (in $V$ ). The exposure time for an image in the chain was 5 minutes. These observations were synchronized with the accuracy of a few seconds. They were carried out in the Orion association (total duration about 35 hours) and in the Pleiades cluster (104 hours). 13 flares in Orion and 38 flares in the Pleiades were detected. Their photographic $B-V$ and $U-B$ indices are in general agreement with those of UV Cet flares (Moffett 1974). The mean $U-B$ index for 45 flares observed near maximum is -0.64 ( $5 \mathrm{~min}$ ), as compared to -0.88 for 153 flares of Moffett's UV Cet stars (1 second time resolution). In both cases, the colours of flare emissions became generally redder after maximum.

\section{Spectra of flare stars}

Originally it was thought that flare (flash) stars in associations and the UV Cet stars also show other differences: the absence of emission lines in the flare star spectra, and their connection with diffuse matter (Herbig 1958, 1968). The former found a natural explanation, the absence of emission lines in flare star spectra was caused by the low spectral resolutions of the objective prism surveys. Spectra of Pleiades flare stars taken with the $6 \mathrm{~m}$-telescope of the Special Astrophysical Observatory showed intense $\mathrm{H}$ and $\mathrm{Ca}$ II emission (Mirzoyan et al. 1990), similar to of UV Cet spectra (Pettersen \& Hawley 1989).

Spectral changes in the flare emission of UV Cet stars were studied by several authors, the most detailed studies being made by Bopp \& Moffett (1973) and Moffett \& Bopp (1976). Using synchronized spectral and photoelectric observations of UV Cet flares with high time resolution, they found spectral changes for flares on different time scales and confirmed Joy \& Humason's (1949) result that during the sharp increase of flare emission the continuum radiation plays the dominant role.

\section{Frequencies of photographically detected flares}

The mean frequency of flares is different for different flare stars. For a given star, it depends on the spectral band and on the flare amplitude, and usually increases with shorter wavelengths and smaller amplitudes. The mean flare frequency of photographic flares is very low compared to that of photoelectric flares of UV Cet stars. A study of the mean flare frequencies of Pleiades flare stars (the duration of photographic observations exceeds 3000 hours) shows that there is a very large range of frequencies: from one flare per some ten hours to one flare per some ten thousand hours (Mirzoyan 1981).

Ambartsumian (1978) developed a statistical method for determining the distribution function of flare frequencies, and applied it to the Pleiades cluster. The number of flare stars with high flare frequencies is larger in the Pleiades than 
in Orion (Parsamian 1980), the number of flare stars with lower flare frequencies is larger in Orion. Ambartsumian (1980) showed that the distribution of mean flare frequencies depends on the luminosities of flare stars. This result has been confirmed for UV Cet stars (Mirzoyan 1981, Mirzoyan \& Hambarian 1988).

\section{Energy spectra of stellar flares}

Changes of evolutionary nature are observed in the energy spectra of flare stars. Gershberg (1972) and Krasnobabtsev \& Gershberg (1975) showed a correlation between energy spectra and luminosities of flare stars, for increasing stellar luminosity the contribution of frequent, less powerful flares decreases. This correlation was confirmed by Lacy et al. (1976) for UV Cet stars. Korotin \& Krasnobabtsev (1986) showed that energy spectra form a natural sequence: energy spectra of UV Cet stars are the continuation of those of flare stars in clusters and associations towards lower energies. This is evidence in favour of a similar physical nature for flare stars of all types.

\section{A possible interpretation of flares. Fast and slow flares}

Knowledge about detailed properties of flares is needed for the study of their physical nature, although the many photographic flare light curves have also proven to be very useful. Haro (1964) noted that all photographically detected flares in clusters and associations can be divided into two groups according to flare rise time. For most flares, the rise time is less than $20-30$ minutes. These are the 'fast' flares. In the second group of 'slow' flares, the rise time is longer than $20-30$ minutes. Haro's division supports Ambartsumian's (1954, 1971) idea about the intrastellar origin of flares: all differences between fast and slow flares (rise time, colour of flare emission, ratio of numbers of flares in the two groups) can be explained by the difference in depth of the layer where the flares originate (corresponding energies are liberated): in the atmosphere of the star or above it. The fast or slow behaviour of a flare does not depend on the physical properties of the flare star on which it occurs. Almost all flare stars showing 'slow' flares have also shown fast flares (Mirzoyan 1981).

In the case of photoelectric flares of UV Cet stars (Moffett 1974) it was found that flare rise times are distributed continuously (Mirzoyan \& Melikian 1986). The correlation between mean flare rise time and luminosity of the flare star is in line with the interpretation of 'fast' and 'slow' flares and also in agreement with the fact that the percentage of 'slow' flares increases towards younger systems, i.e. towards flare stars of higher luminosity.

\section{On the evolution of red dwarf stars}

Photographic observations of flare stars in the Galaxy allow us to outline the evolutionary path of red dwarf stars (Mirzoyan 1990, 1993). The main results were presented by Haro \& Chavira (1965), Haro (1976), and Ambartsumian and Mirzoyan $(1970,1975)$. Here we give some, partially new, observational results, which confirm the evolutionary concept of red dwarf stars. 
1. T Tau and flare stars have physical and dynamical similarities: they coexist in stellar associations (Haro \& Chavira 1965, Rosino 1969); characteristic features of $\mathrm{T}$ Tau stars appear distinctly in flare stars, usually during flares (Ambartsumian 1954); some T Tau stars show flares (Haro \& Chavira 1965, Rosino 1969); multiple stars of the trapezium type are known to consist of combinations of T Tau and flare stars (Hambarian 1988). These results indicate a physical and evolutionary connection between $\mathrm{T}$ Tau and flare stars. However, $\mathrm{T}$ Tau stars belong to the characteristic population of stellar associations, they present the first stage of evolution of red dwarfs after formation.

The coexistence of young flare stars with $\mathrm{T}$ Tau stars in stellar associations, and the existence of comparatively old flare stars in star clusters and the general field where $\mathrm{T}$ Tau stars are absent, show that flare stars represent the next stage of evolution of red dwarfs after the T Tau stars (Haro 1976, Ambartsumian \&Mirzoyan 1970). The question is: is the evolutionary stage of flare stars obligatory for all red dwarf stars?

A positive answer was given by Ambartsumian (1969), who showed that all low luminosity stars in the Pleiades should be flare stars, and concluded that all red dwarf stars pass through this stage of evolution. The discovery of T Tau stars with flare activity showed that both evolutionary stages are overlapping (Ambartsumian 1970). Independent confirmation of the evolutionary status of flare stars was obtained by Mirzoyan et al. (1990): of 34 red dwarf stars studied by Stauffer (1980), 27 turned out to be known flare stars from the catalogue of Haro et al. (1982), and one may suppose that the remaining ones are as yet unknown flare stars.

The high percentage of double and multiple stars among flare stars is noteworthy. Statistics by Gershberg (1978) showed that more than half of the known UV Cet stars are members of multiple systems. Evans (1977) gives a value of about $85 \%$ double stars. Rodono (1979) has shown that double stars occur among flare stars more frequently than among other stars.

2. Flare stars in star clusters and associations and UV Cet stars in the solar vicinity are objects of the same physical class by their observed properties (light curves, colours, spectra, energy spectra, physical nature, etc.) (Mirzoyan 1993). In this context, the space distribution of flare stars of different luminosities in the Galaxy, and the origin of the UV Cet stars of the solar vicinity, provide decisive arguments, which have been studied in detail by Arakelian (1969) and Garibjanian (1976). They conclude that the space density of these stars decreases with the distance from the Sun, in agreement with Ambartsumian's (1957) suggestion that UV Cet stars form a cluster, in which they originated, around the Sun. However, Arakelian's and Garibjanian's conclusion is in contradiction with the large variety of ages and kinematical characteristics of the UV Cet stars (Gershberg 1978, Mirzoyan 1981). It appears now that UV Cet stars of the lowest luminosities are distributed uniformly in the galactic disk (Mirzoyan et al. 1988), after the disintegration of their 'maternal' systems.

The observational data indicate that all flare stars originate in star clusters and associations. Young and middle aged flare stars of comparatively high lu- 
minosity are observed in star clusters and associations where they originated. Their flare activity can be considered as a criterion for their membership in young systems (Mirzoyan et al. 1993). Old flare stars of low luminosity kept their flare activity because of their slow evolution (small masses). They are mainly observed in the general galactic field, after disintegration of their 'maternal' systems. Kunkel (1975) has shown that the duration of flare activity stage for stars of absolute magnitude $M=15$ amounts to some billion years. The majority of flare stars of middle ages are observed in star clusters, and the flare activity of comparatively high luminosity stars can be considered as a criterion for their cluster membership (Mirzoyan et al. 1993).

The observed space distribution of flare stars of different luminosities in the Galaxy is thus a natural consequence of their different ages and the increase of duration of the flare activity stage of evolution towards flare stars of lower luminosities (Mirzoyan 1993).

Haro $(1962,1964,1968)$ and Haro \& Chavira (1965) have shown that in every stellar system there is a boundary spectral class (luminosity) which divides all stars of the system into two groups: flare and non-flare stars. The limit shifts towards later spectral classes (lower luminosities) when older systems are considered. The comparison of flare stars in young (Orion association) and comparatively old (Pleiades cluster) systems shows also that flare stars of the same luminosity are more active in the younger system.

3. The total number of red dwarf stars in the Galaxy is about $10^{10}$ (Mirzoyan 1993). The overwhelming majority is found in the general field and only a small part in clusters and associations.

4. The physical nature of flare events is not completely understood. Even the flares observed in the Sun, which differ from stellar flares in scale and in nature (see, e.g. Mirzoyan 1981 and Lang 1990) have no generally accepted interpretation (Mirzoyan 1993). However, the discovery and study of the 'fast' and 'slow' flares, their similarity with the processes on the $\mathrm{T}$ Tau stars and related objects seem to offer a perspective.

\section{Conclusion}

A number of observatories take part in photographic observations of flare stars in star clusters and associations (Mirzoyan 1981, 1993). Among them, the most active ones were Tonantzintla and Byurakan. Although the photographic method of observations is evidently very coarse when compared with the photoelectric method, it is the most effective one for collecting a large amount of material. Flares detected by photographic observations are generally more energetic than those registered by the photoelectric method. Photographic observations are successfully used for the search of flare stars in star clusters and associations. Without these, the overwhelming majority of results on the evolution of red flare stars would not exist. On the basis of all these observations, the probable evolutionary path of red dwarf stars is as follows (Mirzoyan 1981):

$T$ Tau stars $\rightarrow$ Flare stars $\rightarrow$ red dwarf stars of constant radiation

The possibility to observe all three stages together in the nearest associations is due to the favourable circumstance that evolution progresses more rapidly in 
stars of higher mass (luminosity). This is the first successful attempt to find a complete evolutionary path of stars, on the basis of observations (see, e.g. Ambartsumian 1947, Ambartsumian \& Mirzoyan 1982, Mirzoyan 1984), without any theoretical assumption. After the discovery of stellar associations, which for the first time indicated that star formation in the Galaxy continues to the present time (Ambartsumian 1947, Ambartsumian \& Mirzoyan 1982), the concept of red dwarf evolution (Haro 1976, Ambartsumian \& Mirzoyan 1970) can be considered the most important achievement of the observational approach to early stages of stellar evolution.

Although the fundamental concept (Haro 1957) about the evolutionary status of flare stars seems to be beyond doubt, further studies are needed. It is necessary to organize synchronous observations of young and more luminous flare stars in clusters and associations and old and less luminous UV Cet stars in different spectral regions (optical, far UV, radio, X-ray) using ground based and space observatories as noted by Rodonò (1990). This approach may bring a solution concerning the physical nature of flare events, and maybe of stellar instability in general (Mirzoyan 1991). Let us hope that such cooperation will be realized before the beginning of the XXIth century.

\section{References}

Ambartsumian V.A., 1947, Stellar Evolution and Astrophysics, Acad. Sci. Armenia, Yerevan

Ambartsumian V.A., 1954, Comm. Byurakan Obs. 13, 3

Ambartsumian V.A., 1957, in Non-Stable Stars, M.A. Arakelian (ed.), Acad. Sci. Armenia, Yerevan, p. 9

Ambartsumian V.A., 1969, in Stars, Nebulae, Galaxies, V.V. Sobolev (ed.), Acad. Sci. Armenia, Yerevan, p. 283

Ambartsumian V.A., 1970, Astrofiz. 6, 31

Ambartsumian V.A., 1971, Astrofiz. 7, 557

Ambartsumian V.A., 1978, Astrofiz. 14, 367

Ambartsumian V.A., 1980, in Flare Stars, Fuors and Herbig-Haro Objects, L.V. Mirzoyan (ed.), Acad. Sci. Armenia, Yerevan, p. 85

Ambartsumian V.A., Mirzoyan L.V., 1970, in IAU Coll. No. 15, New Directions and New Frontiers in Variable Star Research, Veröff. Bamberg 9, No. 100, p. 98

Ambartsumian V.A., Mirzoyan L.V., 1975, in IAU Symp. 67, Variable Stars and Stellar Evolution, V. Sherwood and L. Plaut (eds.), Reidel, Dordrecht, p. 3

Ambartsumian V.A., Mirzoyan L.V., 1982, ApSS 84, 317

Arakelian M.A., 1969, in IAU Coll. 4, Non-Periodic Phenomena in Variable Stars, L. Detre (ed.), Academic Press, Budapest, p. 161

Bopp B.W., Moffett T.J., 1973, ApJ 185, 239

Evans D.S., 1977, in Flare Stars, L.V. Mirzoyan (ed.), Acad. Sci. Armenia, Yerevan, p. 40

Garibjanian A.T., 1976, Comm. Byurakan Obs. 49, 63

Gershberg R.E., 1972, ApSS 19, 75

Gershberg R.E., 1978, Flare Stars of Small Masses, Nauka, Moscow

Hambarian V.V., 1988, Astrofiz. 28, 149

Haro G., 1957, in IAU Symp. No. 3, Non-Stable Stars, G.H. Herbig (ed.), Cambridge University Press, Cambridge, p. 26 
Haro G., 1962, in Stellar Evolution, J. Sahade (ed.), Astr. Obs. Univ. La Plata, p. 37

Haro G., 1964, in IAU-URSI Symp. No. 20, The Galaxy and the Magellanic Clouds,

F.J. Kerr and A.W. Rodgers (eds.), Australian Acad. Sci., Canberra, p. 30

Haro G., 1968, in Stars and Stellar Systems, Vol. 7, B.M. Middlehurst and L.H. Aller (eds.), Chicago Univ. Press, Chicago, p. 141

Haro G., 1976, Bol. Inst Tonantzintla 2, 3

Haro G., Chavira, E. 1965, Vistas Astr. 8, 89

Haro G., Morgan W., 1953, ApJ 118, 16

Haro G., Chavira E., Gonzalez G., 1982, Bol. Inst. Tonantzintla 3, No. 1

Herbig G.H., 1958, in Stellar Populations, D.J.K. O'Connell (ed.), North Holland, Amsterdam, p. 127

Herbig G.H., 1968, ApJ 135, 736

Joy A.H., Humason M.L., 1949, PASP 64, 133

Korotin S.A, Krasnobabtsev V.I., 1986, in Flare Stars and Related Objects, L.V. Mirzoyan (ed.), Acad. Sci. Armenia, Yerevan, p. 147

Krasnobabtsev V.I., Gershberg R.E., 1975, Izv. Krym. Astrofiz. Obs. 53, 154

Kunkel W., 1975, in IAU Symp. 67, Variable Stars and Stellar Evolution, V.Sherwood and L. Plaut (eds.), Reidel, Dordrecht, p. 15

Lacy C.H., Moffett T.J., Evans D.S., 1976, ApJS 30, 85

Lang K.R., 1990, in IAU Symp. 137, Flare Stars in Clusters, Associations, and the Solar Vicinity, L.V. Mirzoyan et al. (eds.), Kluwer, Dordrecht, p. 125

Mirzoyan L.V., 1980, in Flare Stars, Fuors and Herbig-Haro Objects, L.V. Mirzoyan (ed.), Acad. Sci. Armenia, Yerevan

Mirzoyan L.V., 1981, Stellar Instability and Evolution, Acad. Sci. Armenia, Yerevan

Mirzoyan L.V., 1984, Vistas Astr. 27, 77

Mirzoyan L.V., 1990, in IAU Symp. 137, Flare Stars in Clusters, Associations, and the Solar Vicinity, L.V. Mirzoyan et al. (eds.), Kluwer, Dordrecht, p. 1

Mirzoyan L.V., 1991, Early Stages of Stellar Evolution, Acad. Sci. Armenia, Yerevan

Mirzoyan L.V.. 1993, Astrofiz. 36, 277

Mirzoyan L.V., Hambarian V.V., 1988, Astrofiz. 28, 375

Mirzoyan L.V., Melikian N.D., 1986, in Flare Stars and Related Objects, L.V. Mirzoyan (ed.), Acad. Sci. Armenia, Yerevan, p. 153

Mirzoyan L.V., Chavushian H.S., Natsvlishvili R.Sh., Ohanian G.B., Hambarian V.V., Garibjanian A.T., 1981, Astrofiz. 17, 197

Mirzoyan L.V., Chavushian H.S., Melikian N.D., Natsvlishvili R.Sh., Ohanian G.B., Hambarian, V.V., Brutian G.H. 1983, Astrofiz. 19, 725

Mirzoyan L.V., Hambarian V.V., Garibjanian A.T., Mirzoyan A.L., 1988, Astrofiz. 29, 44

Mirzoyan L.V., Hambarian V.V., Garibjanian A.T., Mirzoyan A.L., 1990, Astrofiz. 33, 5

Mirzoyan L.V., Hambarian V.V., Mirzoyan A.L., 1993, Astrofiz. 37, No. 3

Moffett T.J., 1974, ApJS 29, 1

Moffett T.J., Bopp B.W., 1976, ApJS 31, 61

Parsamian, E.S. 1980, Astrofiz. 16, 677

Pettersen B.R., Hawley S.L., 1989, A\&A 217, 187

Rodono M., 1979, Flare Active Binary Systems, Catania Obs. preprint

Rodono M., 1990, in IAU Symp. 137, Flare Stars in Clusters, Associations, and the Solar Vicinity, L.V. Mirzoyan et al. (eds.), Kluwer, Dordrecht, p. 371

Rosino L., 1969, in Low Luminosity Stars, S.S. Kumar (ed.), Gordon \& Breach, New York, p. 181

Shakhovskaya N.L., 1995, this volume, p. 61

Stauffer J.R., 1980, AJ 85, 1341 